\title{
Factors Affecting Accuracy of Distance Measurement System Based on Ultrasonic Sensor in Air
}

\author{
Kamal Bhatia, Ashish Pathak
}

\begin{abstract}
Measuring distance between two objects accurately is very difficult task. Generally we have to carry a measuring apparatus with us all for measuring the distance between two objects. Many systems are designed for measuring the distance but they all lack the accuracy, such as ultrasonic sensor based distance measurement unit. Which uses ultrasonic sensor and a microcontroller for measuring the distance between two objects after measuring the distance it displays the information on a display screen connected with the microcontroller. But the measured distance is not accurate because there are many factors which affects the accuracy of the system by affecting the speed of sound in air. In this paper these factor are discussed and what changes are needed for improving the accuracy of system.
\end{abstract}

Keyword: Ultrasonic sensor, sound speed, air, display screen, microcontroller, distance measurement, accuracy.

\section{INTRODUCTION}

The ultrasonic sensors are ideal for measuring distance between two objects without any measurement equipment's. The ultrasonic sensor is used with microcontrollers for measuring the distance between two objects[1]. The microcontroller can be programmed for measuring the distance between two objects[2]. But there are many factor which effects the accuracy of a distance measurement unit such as air density, air pressure, temperature, distance between objects, orientation of object and program used for calculating distance. These all factors changes the speed of sound in both ways i.e. some of these improves the accuracy and some of these decreases the accuracy.

\section{TECHNOLOGY USED}

Arduino Uno: An Arduino Uno is a microcontroller used for controlling components, sensors in a controlled manner, the Arduino Uno have a programmable IC Atmega328P[3], which is programmed by software Arduino IDE installed in users computer unit[4]. The Arduino Uno is an open source hardware, having plurality of digital and analogue pins used for controlling or performing different set of tasks.

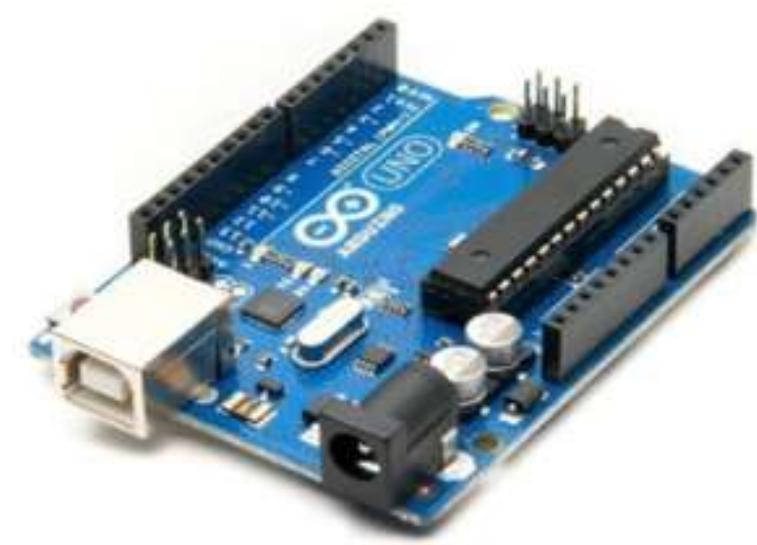

Figure 1: Arduino Uno

HC-SR04: The HC-SR04 is an Ultrasonic sensor which have two different components first is transmitter which transmits sound wave at ultrasonic frequency and second is receiver which receives the ultrasonic sound which got reflected by any object in its path[5]. The HC-SR04 is have four connection pins GND, Vcc, ECHO and TRIGGER pin. The GND pin is ground, Vcc for supply electrical charge to sensor for operation, Echo pin for receiving echo through receiver and sending its data to microcontroller and trigger is used for triggering transmitter unit[6].

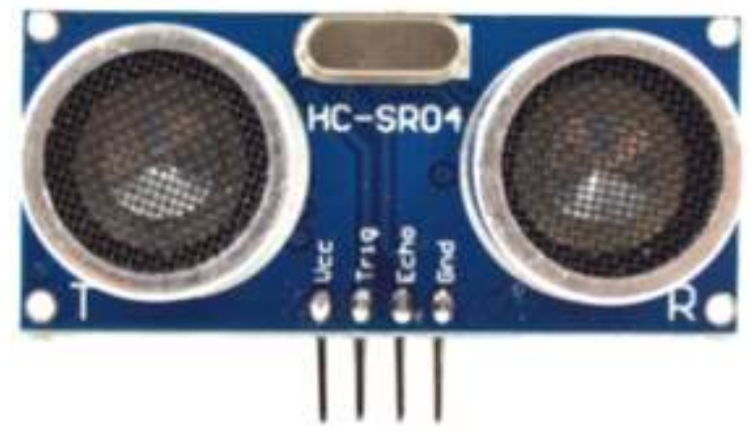

Figure 2: HC-SR 04

Display Unit: The display unit is $16 * 2$ LCD screen which is connected with Arduino Uno for displaying data received from Ultrasonic sensor and processed by Arduino Uno[7].
Revised Version Manuscript Received on 10 September, 2019.

Kamal Bhatia, Department of Electronics \& Communication Engineering, Noida Institute of Engineering and Technology, Noida, U.P. India. (Email: researchnietip@gmail.com)

Ashish Pathak, Department of Physics, Noida Institute of Engineering and Technology, Noida, U.P. India. (Email: researchnietip@gmail.com) 


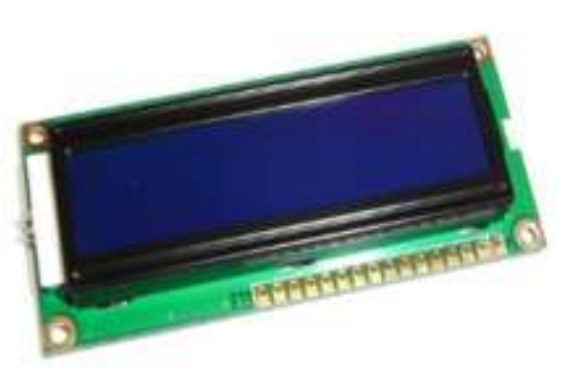

Figure 3: 16*2 LCD Display Screen

\section{SYSTEM DESIGN \& RESULTS}

The system which is designed for testing the factors affecting the accuracy of distance measurement unit contains, a microcontroller, a ultrasonic sensor, a display unit, a physical distance measuring apparatus (a centi-meter scale) and object.

The microcontroller is an Arduino Uno is used for programming and controlling all the components of system. The Arduino Uno is connected with ultrasonic sensor by using connecting wires, the display screen is connected with Arduino Uno by connecting wires. The system is designed and formed upon a breadboard.

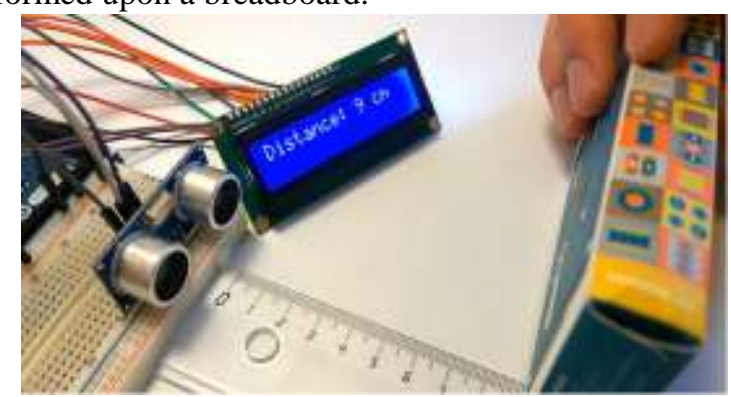

Figure 4: Distance Measurement Unit

Factor Affecting Accuracy of Distance Measurement Unit

These all experiments are performed in controlled climatic conditions in labs and all the results are described here are recorded in controlled climatic conditions.

\section{Effect of Temperature:}

The speed of sound is directly proportional to temperature i.e. when the temperature increases the speed of sound is also increases

$$
\begin{aligned}
& v=\sqrt{\frac{\gamma R T}{\rho V}}=\sqrt{\frac{\gamma R T}{M}} \\
& v \propto \sqrt{T} \\
& v_{t}=(332+0.61 t) \mathrm{m} / \mathrm{s}
\end{aligned}
$$

From the above given equation we can say that the speed of sound increases in multiple of 0.61 as temperature increases for example: if temperature if $2 \mathrm{oC}$ then the speed of sound will be $333.21 \mathrm{~m} / \mathrm{s}$ and if temperature increases by $4 \mathrm{oC}$ then speed of sound will be $334.42 \mathrm{~m} / \mathrm{s}$.

\section{Effect of Density:}

The effect of density is negative on speed of sound i.e. when density increases the speed of sound deceases and will affect the accuracy of distance measurement unit.

$$
v_{\text {air }}=\sqrt{\frac{y P}{\rho}}
$$

From above equation we can say that speed of sound in air is inversely proportion to square root of density of air.

Effect of Pressure:

The pressure does not have any kind of effect on the speed of sound in air.

\section{Effect of humidity}

When humidity increases, number of water molecules also increases thus there is a decrease in molar mass of air, hence speed of sound increases i.e. the speed of sound is directly proportional to humidity.

\section{Effect of Material and its orientation}

The accuracy of distance measurement unit depends is also depends upon material of object and the orientation of the material. When the material of object is sound absorbing it will not reflects the sound back and the receiver of ultrasonic sensor will not receive any data back and the unit will stop working or will show wrong data. The orientation of object should be considered because when an object is orientated in different angle than measurement unit it will deflects the sound wave in different direction and affects the measurement unit will not receive any data back for processing and shows error or nothing on display.

\section{CONCLUSION}

While designing a system for distance measurement we have to consider all the factor such as temperature, humidity, air density, material and orientation of object and implement the sensors accordingly for measuring the changes climatic condition and implementing the changes in microcontroller program according to the data received from the sensors implemented in the system for accurate results of measured distance.

\section{REFERENCES}

1. Carullo and M. Parvis, "An ultrasonic sensor for distance measurement in automotive applications," IEEE Sens. J., 2001.

2. D. R. Tobergte and S. Curtis, "Arduino Uno," J. Chem. Inf. Model., 2013.

3. Atmel, "ATmega328 / P," 2016.

4. D. Wheat and D. Wheat, "Arduino Software," in Arduino Internals, 2012.

5. C. Scott, "HC-SR04 Ultrasonic Sensor," Arduino Basics, 2012 .

6. K. Manpreet and P. Jai, "Distance measurement of object by Ultrasonic Sensor HC-SR04," IJSRD-International J. Sci. Res. Dev., 2015.

7. M. McRoberts, Beginning Arduino. 2013.

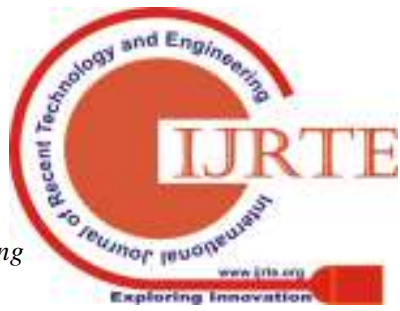

